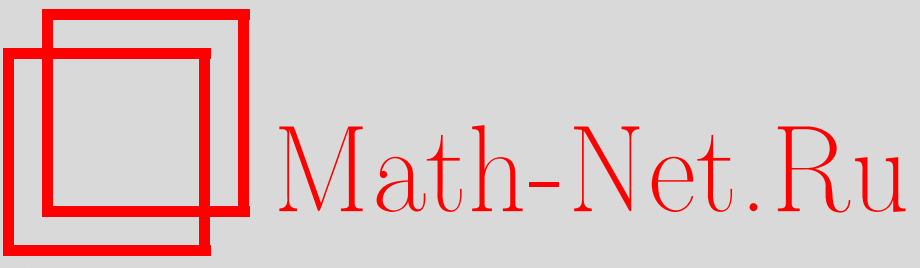

N. V. Pertsev, Stability of linear delay differential equations arising in models of living systems, Mat. Tr., 2019, Volume 22, Number 2, 157-174

DOI: https://doi.org/10.33048/mattrudy.2019.22.209

Use of the all-Russian mathematical portal Math-Net.Ru implies that you have read and agreed to these terms of use

http://www.mathnet.ru/eng/agreement

Download details:

IP : 54.210 .77 .194

April 26, 2023, 18:03:02 


\title{
ОБ УСТОЙЧИВОСТИ РЕШЕНИЙ ЛИНЕЙНЫХ ДИФФЕРЕНЦИАЛЬНЫХ УРАВНЕНИЙ С ЗАПАЗДЫВАНИЕМ, ВОЗНИКАЮЩИХ В МОДЕЛЯХ ЖИВЫХ СИСТЕМ
}

\begin{abstract}
Н. В. Периев
Представлены результаты исследования устойчивости тривиального решения системы линейных дифференциальных уравнений с запаздыванием, разложимой на две подсистемы. Каждая из подсистем содержит матрицы специального вида. Установлены условия асимптотической устойчивости и неустойчивости тривиального решения на основе свойств устойчивых матриц и невырожденных М-матриц. Исследована устойчивость положений равновесия математических моделей в иммунологии и эпидемиологии.

Ключевые слова и фразы: система линейных дифференциальных уравнений с запаздыванием, устойчивость тривиального решения, неотрицательная матрица, устойчивая матрица, М-матрица, системы уравнений Важевского, математические модели в иммунологии и эпидемиологии.
\end{abstract}

\section{Введение}

Дифференциальные уравнения с запаздыванием широко применяются при исследовании различных процессов в технике, экономике, биологии, иммунологии, эпидемиологии и в других областях. Важным этапом изучения систем дифференциальных уравнений является анализ устойчивости их положений равновесия. Один из стандартных подходов к решению этой задачи связан с переходом к системе дифференциальных уравнений линейного приближения и ее последующим анализом. Изучение условий асимптотической устойчивости (неустойчивости) тривиальных решений возникающих систем линейных дифференциальных уравнений с запаздыванием часто наталкивается на значительные затруднения при анализе корней характеристического уравнения или применении функционалов Ляпунова - Красовского. Указанные затруднения, как правило, обусловлены высокой размерностью исследуемых систем дифференциальных уравнений или наличием нескольких запаздываний, включая распределенные запаздывания.

\footnotetext{
Работа выполнена при финансовой поддержке Российского фонда фундаментальных исследований (проект № 18-29-10086).
}

(C) Н. В. Перцев; 2019 
В ряде случаев системы дифференциальных уравнений линейного приближения могут содержать матрицы специального вида. Наличие таких матриц позволяет использовать известные алгебраические критерии, существенно упрощающие решение задачи об устойчивости тривиального решения той или иной системы дифференциальных уравнений. Одни из первых результатов в этом направлении приведены в работах $[6 ; 10 ; 17$; 20]. Применение результатов перечисленных работ к исследованию конкретных математических моделей живых систем представлено, например, в $[6 ; 9 ; 12 ; 13]$.

Целью настоящей статьи является изучение условий устойчивости тривиального положения равновесия систем дифференциальных уравнений с запаздыванием, разложимых на две подсистемы, содержащих матрицы специального вида. Полученные результаты используются для анализа решений математических моделей, возникающих в задачах иммунологии и эпидемиологии.

\section{§1. Постановка задачи}

Рассмотрим систему дифференциальных уравнений с запаздыванием

$$
\frac{d x(t)}{d t}=\sum_{i=0}^{n} C_{i} x\left(t-\omega_{i}\right)+\int_{-\tau}^{0} C_{n+1}(\theta) x(t+\theta) d \theta, \quad t \geqslant 0,
$$

дополненную начальным условием

$$
x(t)=\varphi(t), \quad t \in I_{\omega, \tau}=\left[-\max \left\{\omega_{1}, \ldots, \omega_{n}, \tau\right\} ; 0\right],
$$

где $x(t)=\left(x_{1}(t), \ldots, x_{m}(t)\right)^{T} \in \mathbb{R}^{m}-$ искомая функция, $m \geqslant 2$, символ « $T$ означает операцию транспонирования, константы $\omega_{0}=0,0<\omega_{i}<\infty$, $1 \leqslant i \leqslant n, 0<\tau<\infty, C_{0}, C_{1}, \ldots, C_{n}-m \times m$-матрицы, $C_{n+1}(\theta)-m \times m$ матрица, все элементы которой - интегрируемые по Риману на промежутке $[-\tau ; 0]$ функции, $\varphi(t)=\left(\varphi_{1}(t), \ldots, \varphi_{m}(t)\right)^{T} \in \mathbb{R}^{m}$ - заданная непрерывная функция.

Следуя [8; 14], решением системы (1) с начальным условием (2) на промежутке $[0 ; \infty)$ назовем непрерывную на промежутке $I_{\omega, \tau} \cup[0 ; \infty)$ функцию $x=x(t)$, непрерывно дифференцируемую (покомпонентно) на промежутке $[0 ; \infty)$, удовлетворяющую начальному условию $(2)$ и уравнениям системы (1) для всех $t \in[0 ; \infty)$; при $t=0$ под $d x(t) / d t$ понимаем правостороннюю производную

$$
\begin{aligned}
\frac{d x(0)}{d t} & =\sum_{i=0}^{n} C_{i} x\left(-\omega_{i}\right)+\int_{-\tau}^{0} C_{n+1}(\theta) x(\theta) d \theta \\
& =\sum_{i=0}^{n} C_{i} \varphi\left(-\omega_{i}\right)+\int_{-\tau}^{0} C_{n+1}(\theta) \varphi(\theta) d \theta .
\end{aligned}
$$


Система (1) при нулевых начальных данных (2) ( $\varphi \equiv 0)$ имеет тривиальное решение $x(t) \equiv 0$. Обозначим через $\|v\|_{\mathbb{R}^{m}}$ норму вектора $v \in \mathbb{R}^{m}$ и в соответствии с [8; 14] введем следующие определения.

Тривиальное решение системы (1) называется устойчивым nо Ляnунову, если для любого $\varepsilon>0$ можно указать такое число $\delta=\delta(\varepsilon)>0$, что из неравенства

$$
\max _{t \in I_{\omega, \tau}}\|\varphi(t)\|_{\mathbb{R}^{m}}<\delta
$$

будет следовать неравенство $\|x(t)\|_{\mathbb{R}^{m}}<\varepsilon$ при всех $0 \leqslant t<\infty$. В противном случае тривиальное решение системы (1) называют неустойчивым. Устойчивое тривиальное решение системы (1) называется асимптотически устойчивым по Ляпунову, если для любой функции $\varphi$ из (2) существует $\lim _{t \rightarrow+\infty} x(t)=0$.

Допустим, что систему (1) можно переписать в виде двух подсистем:

$$
\begin{aligned}
& \frac{d z(t)}{d t}=Q z(t)+\sum_{i=0}^{n} D_{i} y\left(t-\omega_{i}\right)+\int_{-\tau}^{0} D_{n+1}(\theta) y(t+\theta) d \theta \\
& \frac{d y(t)}{d t}=\sum_{i=0}^{n} A_{i} y\left(t-\omega_{i}\right)+\int_{-\tau}^{0} A_{n+1}(\theta) y(t+\theta) d \theta-B y(t)
\end{aligned}
$$

где

$$
\begin{gathered}
x(t)=\left(z_{1}(t), \ldots, z_{\ell}(t), y_{1}(t), \ldots, y_{k}(t)\right)^{T}, \quad \ell+k=m, \\
z(t)=\left(z_{1}(t), \ldots, z_{\ell}(t)\right)^{T}, \quad y(t)=\left(y_{1}(t), \ldots, y_{k}(t)\right)^{T},
\end{gathered}
$$

$Q-\ell \times \ell$-матрица, $D_{0}, D_{1}, \ldots, D_{n}-\ell \times k$-матрицы, $A_{0}, A_{1}, \ldots, A_{n}-k \times k$ матрицы, $D_{n+1}(\theta)-\ell \times k$-матрица и $A_{n+1}(\theta)-k \times k$-матрица, все элементы этих матриц представляют собой интегрируемые по Риману на промежутке $[-\tau ; 0]$ функции, $B=\operatorname{diag}\left(b_{11}, \ldots, b_{k k}\right)$ - диагональная матрица. Полагаем, что $Q$ - устойчивая матрица (все ее собственные числа имеют отрицательную действительную часть [1]), все диагональные элементы матрицы $B$ положительны.

Исследуем проблему устойчивости тривиального решения системы (1), опираясь на ее запись в форме системы (3), (4).

\section{§2. Вспомогательные утверждения и основные результаты}

Рассмотрим задачу Коши

$$
u(0)=u_{0} \in \mathbb{R}^{m}, \quad \frac{d u(t)}{d t}=A u(t)+f(t), \quad 0 \leqslant t<\infty,
$$


где $u(t) \in \mathbb{R}^{m}, A-m \times m$-матрица, вектор-функция $f=f(t) \in \mathbb{R}^{m}$ непрерывна на промежутке $[0 ; \infty)$. Решением задачи Коши (5) на промежутке $[0 ; \infty)$ назовем непрерывно дифференцируемую (покомпонентно) на промежутке $[0 ; \infty)$ функцию $u=u(t)$, удовлетворяющую начальному условию и уравнениям системы (5) для всех $t \in[0 ; \infty)$; при $t=0$ под $d u(t) / d t$ понимаем правостороннюю производную

$$
\frac{d u(0)}{d t}=A u_{0}+f(0)
$$

Задача Коши (5) имеет на промежутке $[0 ; \infty)$ единственное решение $u(t)$, и его можно записать в виде

$$
u(t)=e^{A t} u_{0}+\int_{0}^{t} e^{A(t-s)} f(s) d s, \quad 0 \leqslant t<\infty,
$$

где выражения $e^{A t}, e^{A(t-s)}$ означают матричные экспоненты [1; 5].

Пусть далее под нормой вектора $v \in \mathbb{R}^{m}$ понимается выражение

$$
\|v\|_{\mathbb{R}^{m}}=\|v\|_{1, m}=\sum_{i=1}^{m}\left|v_{i}\right|
$$

$\left(L_{1}\right.$ - норма в пространстве $\left.\mathbb{R}^{m}\right)$. Полагаем, что в используемых ниже оценках нормы матриц согласованы с $\|v\|_{1, m}$.

Используя представление решения $u(t)$ в форме (6), приходим к следующему результату.

Лемма 1. Пусть в системе (5) матрица $A$ является устойчивой. Тогда при $t \geqslant 0, t-s \geqslant 0$ справедливы оценки

$$
\left\|e^{A t}\right\| \leqslant b e^{-\alpha t}, \quad\left\|e^{A(t-s)}\right\| \leqslant b e^{-\alpha(t-s)},
$$

где $b>0, \alpha>0$ - некоторые константы. Пусть, кроме того, $f(t)$ такова, что $\|f(t)\|_{\mathbb{R}^{m}} \leqslant f^{(*)}$ для всех $t \in[0 ; \infty)$ и $f(t) \rightarrow 0$ при $t \rightarrow+\infty$. Тогда имеет место оценка

$$
\|u(t)\|_{\mathbb{R}^{m}} \leqslant b \max \left\{\left\|u_{0}\right\|_{\mathbb{R}^{m}}, \frac{f^{(*)}}{\alpha}\right\}, \quad t \in[0 ; \infty)
$$

и существует $\lim _{t \rightarrow+\infty} u(t)=0$.

Доказательство леммы 1 вытекает из результатов работы [5]. 
Лемма 2. Пусть решение $y(t) \equiv 0$ системы (4) асимптотически устойчиво. Тогда тривиальное решение

$$
z(t) \equiv 0, \quad y(t) \equiv 0
$$

системы (3), (4) асимптотически устойчиво; если решение $y(t) \equiv 0$ системы (4) неустойчиво, то тривиальное решение (7) системы (3), (4) неустойчиво.

Доказательство. Покажем устойчивость решения (7). Используя обозначения, принятые для переменных систем (3), (4), получаем, что

$$
\|x(t)\|_{1, m}=\|z(t)\|_{1, \ell}+\|y(t)\|_{1, k}, \quad 0 \leqslant t<\infty .
$$

Оценим слагаемые, входящие в (8). Обратимся к системе (4). Опираясь на условия леммы и определение, находим, что $y(t) \equiv 0$ является устойчивым. Для произвольного (но фиксированного) $\varepsilon_{0}>0$ существует $\delta_{0}>0$ такое, что из неравенства

следует неравенство

$$
\max _{t \in I_{\omega, \tau}}\left\|\varphi_{y}(t)\right\|_{1, k}<\delta_{0}
$$

$$
\|y(t)\|_{1, k}<\varepsilon_{0}, \quad 0 \leqslant t<\infty,
$$

где $\varphi_{y}(t)-$ начальная функция для $y(t)$. Если $\delta_{0}>\varepsilon_{0}$, то можно выбрать $\delta_{1}$ таким, что $0<\delta_{1} \leqslant \varepsilon_{0}<\delta_{0}$, и неравенство (9) будет верным для всех начальных функций $\varphi_{y}(t)$, удовлетворяющих условию

$$
\max _{t \in I_{\omega, \tau}}\left\|\varphi_{y}(t)\right\|_{1, k}<\delta_{1}
$$

Как следствие, получаем, что для указанных $\delta_{1}$ и $\varepsilon_{0}$ верно неравенство

$$
\|y(t)\|_{1, k}<\varepsilon_{0}, \quad t \in I_{\omega, \tau} \cup[0 ; \infty) .
$$

Применим лемму 1 к системе (3). Используя (11), приходим к неравенству

$$
\|z(t)\|_{1, \ell} \leqslant b_{Q} \max \left\{\left\|z_{0}\right\|_{1, \ell}, \frac{f_{0}}{\alpha_{Q}}\right\}, \quad t \in[0 ; \infty),
$$

где $b_{Q}, \alpha_{Q}$ - положительные константы, зависящие только от матрицы $Q$,

$$
f_{0}=\left(\sum_{i=0}^{n}\left\|D_{i}\right\|+\int_{-\tau}^{0}\left\|D_{n+1}(\theta)\right\| d \theta\right) \varepsilon_{0},
$$

$z_{0}$ - начальное значение для переменной $z(t)$. Пусть

$$
\left\|z_{0}\right\|_{1, \ell}<\delta_{2}=\frac{f_{0}}{\alpha_{Q}}
$$


Из (12) с учетом (13) выводим неравенство

$$
\|z(t)\|_{1, \ell} \leqslant \frac{\left(\sum_{i=0}^{n}\left\|D_{i}\right\|+\int_{-\tau}^{0}\left\|D_{n+1}(\theta)\right\| d \theta\right) \varepsilon_{0} b_{Q}}{\alpha_{Q}}, \quad t \in[0 ; \infty) .
$$

Обращаясь к (8) и используя (9), (10), (13) и (14), получаем оценку

$$
\|x(t)\|_{1, m} \leqslant \frac{\alpha_{Q}+\left(\sum_{i=0}^{n}\left\|D_{i}\right\|+\int_{-\tau}^{0}\left\|D_{n+1}(\theta)\right\| d \theta\right) b_{Q}}{\alpha_{Q}} \varepsilon_{0}, \quad 0 \leqslant t<\infty .
$$

Пусть $\varepsilon>0-$ произвольное, но фиксированное число. Положим

$$
\varepsilon_{0}=\frac{\alpha_{Q} \varepsilon}{\alpha_{Q}+\left(\sum_{i=0}^{n}\left\|D_{i}\right\|+\int_{-\tau}^{0}\left\|D_{n+1}(\theta)\right\| d \theta\right) b_{Q}} .
$$

Из $(15)$ с учетом соотношений (12) и (13) при выбранном $\varepsilon_{0}$ следует устойчивость решения (7).

Покажем асимптотическую устойчивость решения (7). Исходя из условий леммы, заключаем, что для любой фиксированной начальной функции $\varphi_{y}(t)$ существует $\lim _{t \rightarrow+\infty} y(t)=0$. Подставляя $y(t)$ в систему $(3)$, приходим к очевидному соотношению

$$
\lim _{t \rightarrow+\infty} \sum_{i=0}^{n} D_{i} y\left(t-\omega_{i}\right)=0 .
$$

Интегральное слагаемое из (3) перепишем в виде

$$
v(t)=\int_{-\tau}^{0} D_{n+1}(\theta) y(t+\theta) d \theta=\int_{t-\tau}^{t} D_{n+1}(s-t) y(s) d s .
$$

Обозначим через $d_{i, j}^{(n+1)}(\theta)$ элементы матрицы $D_{n+1}(\theta)$. Для каждой компоненты $v_{i}(t)$ вектора $v(t)$ с учетом теоремы о среднем имеем

$$
\begin{gathered}
v_{i}(t)=\int_{t-\tau}^{t} \sum_{j=1}^{k} d_{i, j}^{(n+1)}(s-t) y_{j}(s) d s=\sum_{j=1}^{k} \int_{t-\tau}^{t} d_{i, j}^{(n+1)}(s-t) y_{j}(s) d s \\
=\sum_{j=1}^{k} y_{j}\left(t_{i, j}^{(*)}\right) \int_{t-\tau}^{t} d_{i, j}^{(n+1)}(s-t) d s=\sum_{j=1}^{k} y_{j}\left(t_{i, j}^{(*)}\right) \int_{-\tau}^{0} d_{i, j}^{(n+1)}(\theta) d \theta, \\
t_{i, j}^{(*)} \in(t-\tau ; t), \quad 1 \leqslant j \leqslant k, \quad 1 \leqslant i \leqslant \ell .
\end{gathered}
$$

Следовательно, для вектора $v(t)$, заданного формулой (17), существует

$$
\lim _{t \rightarrow+\infty} v(t)=0 .
$$


Применяя к системе (3) лемму 1 с учетом (16) и (18), получаем, что для каждого фиксированного $z_{0}$ верна сходимость $z(t) \rightarrow 0$ при $t \rightarrow+\infty$. В итоге имеем, что существует $\lim _{t \rightarrow+\infty} x(t)=0$.

Допустим, что решение $y(t) \equiv 0$ системы (4) неустойчиво. Используя неравенство

$$
\|x(t)\|_{1, m} \geqslant\|y(t)\|_{1, k}, \quad 0 \leqslant t<\infty,
$$

и определение, получаем, что тривиальное решение (7) системы (3), (4) является неустойчивым.

Обратимся к системе (4) и, следуя [8], будем искать ее решения в форме

$$
y(t)=e^{r t} c,
$$

где $r$ - некоторое комплексное число, $c \in \mathbb{R}^{k}-$ ненулевой вектор. Введем матрицу

$$
S(r)=r I+B-\sum_{i=0}^{n} A_{i} e^{-r \omega_{i}}-\int_{-\tau}^{0} A_{n+1}(\theta) e^{r \theta} d \theta,
$$

где $I$ - единичная матрица. Подставляя (19) в систему (4), приходим к следующему характеристическому уравнению для нахождения $r$ :

$$
\operatorname{det} S(r)=0 .
$$

Пусть все корни $r_{j}$ уравнения (20) удовлетворяют неравенству

$$
\operatorname{Re}\left(r_{j}\right)<0 .
$$

Опираясь на [8], получаем, что неравенство (21) является необходимым и достаточным условием для асимптотической устойчивости тривиального решения $y(t) \equiv 0$ системы $(4)$.

Следуя $[2 ; 3 ; 16]$, введем несколько определений и утверждений. Пусть $S=\left(s_{i j}\right)$ - вещественная $m \times m$-матрица, $S^{T}$ - транспонированная матрица, $S^{+}=\left(\left|s_{i j}\right|\right)$. Для $v \in \mathbb{R}^{m}$ пусть неравенство $v>0$ равносильно тому, что все компоненты $v$ положительны, неравенство $v \geqslant 0$ - тому, что все компоненты $v$ неотрицательны. Будем говорить, что матрица $S$ является неотрицательной, если все ее элементы неотрицательны. Матрицу $S$ будем называть квазинеотрицательной, если $s_{i j} \geqslant 0$ для всех $i \neq j$, $1 \leqslant i, j \leqslant m$. Матрицу $S$ с элементами $s_{i j} \leqslant 0$ для всех $i \neq j, 1 \leqslant i, j \leqslant m$, назовем невырожденной М-матрищей, если она не вырождена и матрица $S^{-1}$ неотрицательна.

Пусть $S$ квазинеотрицательна. Известно [3], что устойчивость $S$ эквивалентна выполнению критерия Севастьянова - Котелянского: для каждого углового минора $M_{k}$ матрицы $S$ верно $(-1)^{k} M_{k}>0,1 \leqslant k \leqslant m$.

Пусть $S$ такова, что $s_{i j} \leqslant 0$ для всех $i \neq j, 1 \leqslant i, j \leqslant m$. Тогда следующие утверждения эквивалентны $[2 ; 16]$ : 
1) $S$ является невырожденной М-матрицей;

2) все угловые миноры $S$ положительны;

3) матрица $(-S)$ удовлетворяет критерию Севастьянова - Котелянского;

4) существует $\xi \in \mathbb{R}^{m}, \xi>0$, такой, что $S \xi>0$;

5) существует $\psi \in \mathbb{R}^{m}, \psi>0$, такой, что $S^{T} \psi>0$.

Лемма 3. Допустим, что матрицы $A_{0}, A_{1}, \ldots, A_{n}, A_{n+1}(\theta)$, входящие в (4), являются неотрицательными. Обозначим

$$
G_{\Sigma}=B-\sum_{i=0}^{n} A_{i}-\int_{-\tau}^{0} A_{n+1}(\theta) d \theta .
$$

Для асимптотической устойчивости тривиального решения $y(t) \equiv 0$ системы (4) необходимо и достаточно, чтобы матрица $\left(-G_{\Sigma}\right)$ удовлетворяла критерию Севастьянова - Котелянского или, что равносильно, чтобы $G_{\Sigma}$ являлась невырожденной М-матрицей.

Лемма 4. Пусть хотя бы одна из матриц $A_{0}, A_{1}, \ldots, A_{n}, A_{n+1}(\theta)$, входящих в (4), отлична от неотрицательной матрицы. Положим

$$
G_{\Sigma}^{+}=B-\sum_{k=0}^{n} A_{k}^{+}-\int_{-\tau}^{0} A_{n+1}^{+}(\theta) d \theta .
$$

Если матрица $\left(-G_{\Sigma}^{+}\right)$удовлетворяет критерию Севастьянова - Котелянского или, что равносильно, $G_{\Sigma}^{+}$является невырожденной М-матрицей, то тривиальное решение $y(t) \equiv 0$ системы (4) асимптотически устойчиво.

Справедливость леммы 3 вытекает из результатов работы [10], леммы 4 - из результатов работ [6; 20] (с учетом свойств указанных выше матриц специального вида). Отметим, что в условиях леммы 3 системы вида (4) часто называют системами уравнений Важевского.

Лемма 5. Пусть выполнено предположение леммы 3 и $\operatorname{det} G_{\Sigma}<0$. Тогда уравнение (20) имеет вещественный корень $r>0$.

Доказательство. Обратимся к вещественным корням $r$ уравнения (20). Обозначим $P(r)=\operatorname{det} S(r), r \in \mathbb{R}$. Нетрудно заметить, что

$$
P(0)=\operatorname{det} S(0)=\operatorname{det} G_{\Sigma} .
$$

Из условия леммы следует, что $P(0)<0$. При достаточно больших $r>0$ имеем $P(r) \geqslant r^{k}+$ const. Учитывая непрерывность $P(r)$, получаем, что уравнение $P(r)=0$ имеет корень $r_{1}>0$. 
Заметим, что леммы 3-5 не требуют изучения корней уравнения (20) и проверки выполнимости условия (21).

Теорема 1. Пусть хотя бы одна из матриц $G_{\Sigma}, G_{\Sigma}^{+}$, введенных в леммах 3 и 4, является невырожденной М-матрицей. Тогда тривиальное решение (7) системы (3), (4) будет асимптотически устойчивым.

Доказательство. В условиях теоремы применима одна из лемм $3,4$. Отсюда следует, что решение $y(t) \equiv 0$ системы (4) асимптотически устойчиво. Используя лемму 2, завершаем доказательство.

Теорема 2. Пусть в системе (4) матрицы $A_{0}, A_{1}, \ldots, A_{n}$ являются неотрицательными, $A_{n+1}(\theta) \equiv 0$. Предположим, что

$$
\operatorname{det}\left(B-\sum_{i=0}^{n} A_{i}\right)<0 .
$$

Тогда тривиальное решение (7) системы (3), (4) неустойчиво.

Доказательство. Опираясь на условия теоремы, применим лемму 5 , учитывая, что

$$
S(r)=r I+B-\sum_{i=0}^{n} A_{i} e^{-r \omega_{i}} .
$$

Получаем, что характеристическое уравнение (20) имеет вещественный корень $r>0$. Из [15] следует, что решение $y(t) \equiv 0$ системы (4) (с учетом $\left.A_{n+1}(\theta) \equiv 0\right)$ является неустойчивым. Используя лемму 2 , завершаем доказательство.

Замечание 1. Предположим, что матрица $A_{n+1}(\theta)$ в условиях теоремы 2 неотрицательна и отлична от нулевой матрицы. Очевидно, что характеристическое уравнение $(20)$ и в этом случае имеет вещественный корень $r>0$. Вместе с тем здесь нельзя гарантировать неустойчивость решения $y(t) \equiv 0$ системы (4). Как указано в [8], ряд, составленный из решений вида (19), не обязательно сходится, и требуется проведение дополнительного исследования.

Замечание 2. Допустим, что в условиях леммы 4 матрица $G_{\Sigma}^{+}$не является невырожденной М-матрицей. Тогда теоремы 1 и 2 не применимы. Для нахождения условий асимптотической устойчивости (неустойчивости) решения $y(t) \equiv 0$ системы (4) следует использовать метод, связанный с построением функционалов Ляпунова - Красовского. Если матрицы, входящие в систему (4), имеют произвольную структуру, то здесь применимы теоремы только общего вида [8; 14; 15]. В ряде случаев можно использовать функционалы Ляпунова - Красовского, учитывающие структуру системы уравнений (4). Некоторые подходы к построению таких функционалов приведены в $[4 ; 7 ; 18 ; 19]$. 


\section{§3. Примеры}

Ниже представлены результаты исследования двух моделей, возникающих в задачах иммунологии и эпидемиологии.

3.1. Устойчивость тривиального решения модели, описывающей динамику ВИЧ-1 инфекиии в организме человека. Рассмотрим систему дифференциальных уравнений с тремя запаздываниями, дополненную интегральными соотношениями и начальными данными:

$$
\begin{aligned}
& \frac{d T(t)}{d t}=r_{T}-\mu_{T} T(t)-\left(\gamma_{T, V} V(t)+\gamma_{T, I} I(t)\right) T(t), \\
& \frac{d I(t)}{d t}=-\left(\mu_{I}+\sigma_{U} \nu_{U}\right) I(t)-\gamma_{I, E} I(t) E(t) \\
& +e^{-\mu_{C} \omega_{C}}\left(\gamma_{T, V} V\left(t-\omega_{C}\right)+\gamma_{T, I} I\left(t-\omega_{C}\right)\right) T\left(t-\omega_{C}\right), \\
& \frac{d V(t)}{d t}=-\mu_{V} V(t)-\gamma_{T, V} T(t) V(t)+e^{-\mu_{U} \omega_{U}} \nu_{U} I\left(t-\omega_{U}\right) \text {, } \\
& \frac{d E(t)}{d t}=-\mu_{E} E(t)+n_{E} \nu_{K} I\left(t-\omega_{K}\right), \quad t \geqslant 0, \\
& C(t)=\int_{t-\omega_{C}}^{t} e^{-\mu_{C}(t-s)}\left(\gamma_{T, V} V(s)+\gamma_{T, I} I(s)\right) T(s) d s, \\
& U(t)=\int_{t-\omega_{U}}^{t} e^{-\mu_{U}(t-s)} \nu_{U} I(s) d s, \\
& K(t)=\int_{t-\omega_{K}}^{t} \nu_{K} I(s) d s, \\
& T(t)=T_{0}(t), \quad I(t)=I_{0}(t), \quad V(t)=V_{0}(t), \quad E(0)=E_{0}, \\
& t \in\left[-\max \left\{\omega_{C}, \omega_{U}, \omega_{K}\right\} ; 0\right] .
\end{aligned}
$$

В модели (23)-(30) принято, что динамика ВИЧ-1 инфекции в организме человека описывается в терминах следующих компонентов:

$V$ - зрелые вирусные частиц (вирионы);

$U$ - незрелые вирусные частицы;

$T$ - клетки-мишени для вирионов;

$C$ - инфицированные клетки (клетки, активированные к производству вирусных частиц);

I - продуктивно-инфицированные клетки (клетки, производящие вирусные частицы);

$E$ - лимфоциты-эффекторы (киллеры);

$K$ - клетки-предшественники лимфоцитов-эффекторов. 
Численности указанных компонентов в момент времени $t$ обозначены соответственно через $V(t), U(t), T(t), C(t), I(t), E(t), K(t)$.

Все параметры системы $(23)-(30)$ считаются положительными. Параметр $r_{T}$ задает скорость поступления клеток $T$ из клеток костного мозга. Параметры $\mu_{T}, \mu_{I}, \mu_{C}, \mu_{V}, \mu_{U}, \mu_{E}$ означают интенсивности гибели рассматриваемых компонентов за счет естественной смертности в расчете на одну клетку или вирусную частицу. Параметр $\nu_{U}$ задает интенсивность производства частиц $U$ в расчете на одну клетку $I$. Параметр $0<\sigma_{U}<1$ отражает долю клеток $I$, которые погибают после производства частиц $U$ (как результат разрушительных процессов отпочковывания частиц $U$ с мембраны клеток $I)$. Произведение $\sigma_{U} \nu_{U}$ означает интенсивность гибели клеток $I$ (в расчете на одну клетку) вследствие указанных разрушительных процессов. Параметр $\nu_{K}$ задает интенсивность стимуляции выработки клеток $K$ за счет опосредованного воздействия на иммунокомпетентные клетки со стороны клеток $I$ (в расчете на одну клетку). Параметр $n_{E}-$ среднее число потомков одной стимулированной к размножению клетки $K$. Параметры $\gamma_{T, V}, \gamma_{T, I}, \gamma_{I, E}$ означают интенсивности взаимодействия клеток соответственно на одну пару $(T, V),(T, I),(I, E)$. Запаздывания $\omega_{U}, \omega_{C}$ отражают продолжительности «созревания» частиц $U$ и клеток $C$; запаздывание $\omega_{K}-$ продолжительность процесса размножения стимулированных клеток $K$.

Функции, входящие в (30), считаются неотрицательными и непрерывными, константа $E_{0}$ неотрицательна. Уравнения $(27)-(29)$ имеют вспомогательный характер и используются для учета баланса численности клеток и вирусных частиц.

Опираясь на [11], получаем, что система (23)-(26), дополненная начальными условиями (30), имеет единственное решение на промежутке $[0 ; \infty)$ и, кроме того, компоненты решения являются неотрицательными. Как следствие, функции $C(t), U(t), K(t)$, заданные формулами (27)-(29), определены, непрерывны и неотрицательны на промежутке $[0 ; \infty)$.

Уравнения (23)-(26) имеют тривиальное положение равновесия

$$
S^{*}=\left(T^{*}, I^{*}, V^{*}, E^{*}\right)=\left(r_{T} / \mu_{T}, 0,0,0\right)
$$

которое интерпретируется как состояние здорового (не инфицированного) человека. Исследуем устойчивость $S^{*}$, опираясь на систему дифференциальных уравнений линейного приближения в окрестности $S^{*}$. В линеаризованной системе сохраним исходные обозначения переменных. Отброшенные нелинейные члены представляют собой произведения переменных, включая переменные с запаздыванием. Вектор отброшенных при линеаризации членов удовлетворяет необходимому условию малости [15]. 
Систему уравнений линейного приближения запишем в блочном виде

$$
\begin{aligned}
& \frac{d T(t)}{d t}=-\mu_{T} T(t)-\left(\gamma_{T, V} V(t)+\gamma_{T, I} I(t)\right) T^{*}, \\
& \frac{d E(t)}{d t}=-\mu_{E} E(t)+n_{E} \nu_{K} I\left(t-\omega_{K}\right), \\
& \frac{d I(t)}{d t}=-\left(\mu_{I}+\sigma_{U} \nu_{U}\right) I(t)+e^{-\mu_{C} \omega_{C}}\left(\gamma_{T, V} V\left(t-\omega_{C}\right)+\gamma_{T, I} I\left(t-\omega_{C}\right)\right) T^{*}, \\
& \frac{d V(t)}{d t}=-\left(\mu_{V}+\gamma_{T, V} T^{*}\right) V(t)+e^{-\mu_{U} \omega_{U}} \nu_{U} I\left(t-\omega_{U}\right) .
\end{aligned}
$$

Структура уравнений (31)-(34) соответствует форме системы (3), (4). Для (31), (32) можно записать так: $Q=\operatorname{diag}\left(-\mu_{T},-\mu_{E}\right)$, остальные матрицы выписываются аналогично. Система (33), (34) содержит диагональную матрицу

$$
B=\operatorname{diag}\left(\mu_{I}+\sigma_{U} \nu_{U}, \mu_{V}+\gamma_{T, V} T^{*}\right),
$$

остальные матрицы имеют неотрицательные элементы. В итоге находим, чTO

$$
G_{\Sigma}=\left(\begin{array}{cc}
\mu_{I}+\sigma_{U} \nu_{U}-e^{-\mu_{C} \omega_{C}} \gamma_{T, I} T^{*} & -e^{-\mu_{C} \omega_{C}} \gamma_{T, V} T^{*} \\
-e^{-\mu_{U} \omega_{U}} \nu_{U} & \mu_{V}+\gamma_{T, V} T^{*}
\end{array}\right)
$$

Применим теоремы 1 и 2. Проверим выполнение неравенств $M_{1}>0$, $M_{2}>0$ для угловых миноров $G_{\Sigma}$. Имеем

$$
M_{1}=\mu_{I}+\sigma_{U} \nu_{U}-e^{-\mu_{C} \omega_{C}} \gamma_{T, I} T^{*}, \quad M_{2}=\operatorname{det} G_{\Sigma} .
$$

Условия выполнения неравенств $M_{1}>0, M_{2}>0$ можно выразить в терминах показателя

$$
R_{0}=\frac{\gamma_{T, I} T^{*} e^{-\mu_{C} \omega_{C}}}{\mu_{I}+\sigma_{U} \nu_{U}}+\frac{\nu_{U} \gamma_{T, V} T^{*} e^{-\left(\mu_{C} \omega_{C}+\mu_{U} \omega_{U}\right)}}{\left(\mu_{I}+\sigma_{U} \nu_{U}\right)\left(\mu_{V}+\gamma_{T, V} T^{*}\right)},
$$

который называется базовым репродуктивным числом. В частности,

$$
\operatorname{det} G_{\Sigma}=\left(\mu_{I}+\sigma_{U} \nu_{U}\right)\left(\mu_{V}+\gamma_{T, V} T^{*}\right)\left(1-R_{0}\right) \text {. }
$$

Опираясь на теоремы 1,2 и учитывая выражения для $M_{1}, M_{2}$, приходим к выводу:

$1)$ если $R_{0}<1$, то положение равновесия $S^{*}$ асимптотически устойчиво;

2) при $R_{0}>1$ положение равновесия $S^{*}$ неустойчиво.

Отметим, что случай $R_{0}=1$ требует отдельного изучения.

Результаты исследования системы (23)-(30) переносятся на модели динамики ВИЧ-1 инфекции с более высокой размерностью и более сложной структурой правых частей уравнений. 
3.2. Устойчивость тривиального решения модели распространения туберкулеза между двумя регионами. Пусть взрослое население двух регионов представлено в виде групп $S_{1}, I_{1}, C_{1}, S_{2}, I_{2}, C_{2}$, которые включают в себя восприимчивых $\left(S_{i}\right)$, инфицированных микобактериями туберкулеза $\left(I_{i}\right)$ и больных туберкулезом $\left(C_{i}\right)$ индивидуумов конкретного региона, $i=1,2$. Обозначим через $S_{i}(t), I_{i}(t), C_{i}(t)$ численности указанных групп в момент времени $t, i=1,2$. Система уравнений модели имеет вид

$$
\begin{aligned}
\frac{d S_{1}(t)}{d t}=- & \beta_{1} S_{1}(t) C_{1}(t)-\left(\lambda_{11}+\lambda_{12}\right) S_{1}(t)+\lambda_{21} S_{2}(t) \\
& +\rho_{1} \exp \left(-\int_{0}^{\tau} \varphi_{1}(s) C_{1}(t+s-\tau) d s\right) \\
\frac{d I_{1}(t)}{d t}= & \left(1-p_{1}\right) \beta_{1} S_{1}(t) C_{1}(t)-\left(\lambda_{11}+\lambda_{12}\right) I_{1}(t)-\left(\gamma_{1}+\alpha_{1} C_{1}(t)\right) I_{1}(t) \\
+ & \eta_{1} C_{1}(t)+\lambda_{21} I_{2}(t)+\rho_{1}\left(1-\exp \left(-\int_{0}^{\tau} \varphi_{1}(s) C_{1}(t+s-\tau) d s\right)\right) \\
\frac{d C_{1}(t)}{d t}= & p_{1} \beta_{1} S_{1}(t) C_{1}(t)-\left(\eta_{1}+\pi_{11}+\pi_{12}\right) C_{1}(t) \\
& +\left(\gamma_{1}+\alpha_{1} C_{1}(t)\right) I_{1}(t)+\pi_{21} C_{2}(t), \\
\frac{d S_{2}(t)}{d t}= & -\beta_{2} S_{2}(t) C_{2}(t)-\left(\lambda_{22}+\lambda_{21}\right) S_{2}(t)+\lambda_{12} S_{1}(t) \\
& +\rho_{2} \exp \left(-\int_{0}^{\tau} \varphi_{2}(s) C_{2}(t+s-\tau) d s\right), \quad t \geqslant 0 \\
\frac{d I_{2}(t)}{d t}= & \left(1-p_{2}\right) \beta_{2} S_{2}(t) C_{2}(t)-\left(\lambda_{22}+\lambda_{21}\right) I_{2}(t)-\left(\gamma_{2}+\alpha_{2} C_{2}(t)\right) I_{2}(t) \\
+ & \eta_{2} C_{2}(t)+\lambda_{12} I_{1}(t)+\rho_{2}\left(1-\exp \left(-\int_{0}^{\tau} \varphi_{2}(s) C_{2}(t+s-\tau) d s\right)\right) \\
\frac{d C_{2}(t)}{d t}= & p_{2} \beta_{2} S_{2}(t) C_{2}(t)-\left(\eta_{2}+\pi_{22}+\pi_{21}\right) C_{2}(t) \\
& +\left(\gamma_{2}+\alpha_{2} C_{2}(t)\right) I_{2}(t)+\pi_{12} C_{1}(t), \\
S_{i}(0)= & S_{i}^{0}, \quad I_{i}(0)=I_{i}^{0}, \quad C_{i}(t)=C_{i}^{0}(t), \quad t \in[-\tau ; 0], i=1,2
\end{aligned}
$$

Опишем функции и параметры, входящие в уравнения (35)-(40). Зафиксируем $i=1,2$. Константа $\rho_{i}>0$ означает постоянную скорость пополнения групп $S_{i}$ и $I_{i}$ за счет взросления молодых индивидуумов, доживших до возраста $\tau$. Функция $\varphi_{i}(s)$ описывает интенсивность инфицирования молодых индивидуумов в зависимости от их возраста $0 \leqslant s \leqslant \tau$. Полагаем, что $\varphi_{i}(s)$ определена, неотрицательна и непрерывна на промежутке 
$s \in[0 ; \tau]$. Параметры $\beta_{i}>0, \alpha_{i}>0$ задают интенсивности контактов индивидуумов групп $S_{i}$ и $I_{i}$ с индивидуумами группы $C_{i}$. При $i \neq j$ параметры $\lambda_{i j} \geq 0$ означают интенсивности переходов индивидуумов групп $S_{i}$ и $I_{i}$ одного региона в такие же группы другого региона вследствие миграционных процессов; аналогичный смысл имеют параметры $\pi_{i j} \geq 0$ для индивидуумов группы $C_{i}, j=1,2$. Параметры $\lambda_{i i}>0, \pi_{i i}>0$ задают интенсивности естественной смертности индивидуумов групп $S_{i}, I_{i}, C_{i}$ соответственно, а также учитывают эмиграцию индивидуумов этих групп в регионы, не рассматриваемые в модели. Кроме того, $\pi_{i i}$ включает в себя интенсивность гибели индивидуумов группы $C_{i}$ от туберкулеза. Константа $0<p_{i}<1$ отражает долю индивидуумов группы $S_{i}$, у которых заболевание развивается сразу же после их инфицирования. Параметр $\gamma_{i}>0$ означает интенсивность спонтанного развития заболевания для индивидуумов группы $I_{i} ; \eta_{i}>0$ - интенсивность выздоровления индивидуумов группы $C_{i}$ вследствие самолечения или лечения, получаемого в медицинских учреждениях. В (41) принято, что $S_{i}^{0} \geqslant 0, I_{i}^{0} \geqslant 0$, а начальные функции $C_{i}^{0}(t)$ определены, неотрицательны и непрерывны на промежутке $t \in[-\tau ; 0]$, $i=1,2$.

Используя [11], заключаем, что система (35)-(40), дополненная начальными условиями (41), имеет единственное решение на промежутке $[0 ; \infty)$ и, кроме того, компоненты решения являются неотрицательными.

Уравнения (35)-(40) имеют тривиальное положение равновесия

$$
S^{*}=\left(S_{1}^{*}, I_{1}^{*}, C_{1}^{*}, S_{2}^{*}, I_{2}^{*}, C_{2}^{*}\right)=\left(S_{1}^{*}, 0,0, S_{2}^{*}, 0,0\right),
$$

где $S_{1}^{*}>0, S_{2}^{*}>0$ удовлетворяют системе линейных уравнений

$$
\begin{aligned}
\left(\lambda_{11}+\lambda_{12}\right) S_{1}^{*}-\lambda_{21} S_{2}^{*} & =\rho_{1}, \\
-\lambda_{12} S_{1}^{*}+\left(\lambda_{22}+\lambda_{21}\right) S_{2}^{*} & =\rho_{2} .
\end{aligned}
$$

Существование, единственность и положительность компонент решения системы (43), (44) является следствием того, что матрица

$$
P=\left(\begin{array}{cc}
\lambda_{11}+\lambda_{12} & -\lambda_{21} \\
-\lambda_{12} & \lambda_{22}+\lambda_{21}
\end{array}\right)
$$

этой системы является невырожденной М-матрицей. В самом деле, если задать $\psi=(1,1)^{T}$, то $\psi>0$ и $P^{T} \psi>0$. Положение равновесия $S^{*}$ интерпретируется как численность населения двух регионов в условиях полного отсутствия заболевших и инфицированных индивидуумов.

Исследуем устойчивость $S^{*}$, опираясь на систему дифференциальных уравнений линейного приближения в окрестности $S^{*}$. 
Воспользуемся известным соотношением

$$
1-e^{-\alpha} \sim \alpha, \quad \alpha \rightarrow 0,
$$

позволяющим преобразовать выражения

$$
\begin{aligned}
\exp & \left(-\int_{0}^{\tau} \varphi_{i}(s) C_{i}(t+s-\tau) d s\right), \\
1-\exp & \left(-\int_{0}^{\tau} \varphi_{i}(s) C_{i}(t+s-\tau) d s\right),
\end{aligned} \quad i=1,2,
$$

входящие в уравнения (35), (36), (38), (39) изучаемой системы. Вектор отброшенных при линеаризации членов удовлетворяет необходимому условию малости [8]. В линеаризованной системе сохраним исходные обозначения переменных.

Систему уравнений линейного приближения запишем в блочном виде

$$
\begin{aligned}
\frac{d S_{1}(t)}{d t}=- & \left(\lambda_{11}+\lambda_{12}\right) S_{1}(t)+\lambda_{21} S_{2}(t)-\beta_{1} S_{1}^{*} C_{1}(t) \\
& -\rho_{1} \int_{0}^{\tau} \varphi_{1}(s) C_{1}(t+s-\tau) d s \\
\frac{d S_{2}(t)}{d t}=\lambda_{11} S_{1}(t)-\left(\lambda_{22}+\lambda_{21}\right) S_{2}(t)-\beta_{2} S_{2}^{*} C_{2}(t) & -\rho_{2} \int_{0}^{\tau} \varphi_{2}(s) C_{2}(t+s-\tau) d s \\
\frac{d I_{1}(t)}{d t}=- & \left(\lambda_{11}+\lambda_{12}+\gamma_{1}\right) I_{1}(t)+\left(\eta_{1}+\left(1-p_{1}\right) \beta_{1} S_{1}^{*}\right) C_{1}(t) \\
& +\rho_{1} \int_{0}^{\tau} \varphi_{1}(s) C_{1}(t+s-\tau) d s+\lambda_{21} I_{2}(t), \\
\frac{d C_{1}(t)}{d t}=- & \left(\eta_{1}+\pi_{11}+\pi_{12}\right) C_{1}(t)+p_{1} \beta_{1} S_{1}^{*} C_{1}(t)+\gamma_{1} I_{1}(t)+\pi_{21} C_{2}(t), \\
\frac{d I_{2}(t)}{d t}=- & \left(\lambda_{22}+\lambda_{21}+\gamma_{2}\right) I_{2}(t)+\left(\eta_{2}+\left(1-p_{2}\right) \beta_{2} S_{2}^{*}\right) C_{2}(t) \\
& +\rho_{2} \int_{0}^{\tau} \varphi_{2}(s) C_{2}(t+s-\tau) d s+\lambda_{12} I_{1}(t), \\
\frac{d C_{2}(t)}{d t}=- & \left(\eta_{2}+\pi_{22}+\pi_{21}\right) C_{2}(t)+p_{2} \beta_{2} S_{2}^{*} C_{2}(t)+\pi_{12} C_{1}(t)+\gamma_{2} I_{2}(t) .
\end{aligned}
$$

Структура уравнений (45)-(50) соответствует форме системы (3), (4). Для уравнений $(45)$ и $(46)$ имеем, что $Q=(-P)$ - устойчивая матрица. Остальные матрицы, входящие в (45) и (46) выписываются аналогично. Система (47)-(50) содержит диагональную матрицу

$$
B=\operatorname{diag}\left(\lambda_{11}+\lambda_{12}+\gamma_{1}, \eta_{1}+\pi_{11}+\pi_{12}, \lambda_{22}+\lambda_{21}+\gamma_{2}, \eta_{2}+\pi_{22}+\pi_{21}\right),
$$


элементы остальных матриц неотрицательны. Выполняя промежуточные выкладки, получаем

$$
\begin{gathered}
G_{\Sigma}=\left(\begin{array}{cccc}
g_{11} & -g_{12} & -g_{13} & 0 \\
-g_{21} & g_{22} & 0 & -g_{24} \\
-g_{31} & 0 & g_{33} & -g_{34} \\
0 & -g_{42} & -g_{43} & g_{44}
\end{array}\right), \\
g_{11}=\lambda_{11}+\lambda_{12}+\gamma_{1}, \quad g_{12}=\eta_{1}+\left(1-p_{1}\right) \beta_{1} S_{1}^{*}+\rho_{1} J_{1}, \quad g_{13}=\lambda_{21}, \\
g_{21}=\gamma_{1}, \quad g_{22}=\eta_{1}+\pi_{11}+\pi_{12}-p_{1} \beta_{1} S_{1}^{*}, \quad g_{24}=\pi_{21}, \\
g_{31}=\lambda_{12}, \quad g_{33}=\lambda_{22}+\lambda_{21}+\gamma_{2}, \quad g_{34}=\eta_{2}+\left(1-p_{2}\right) \beta_{2} S_{2}^{*}+\rho_{2} J_{2}, \\
g_{42}=\pi_{12}, \quad g_{43}=\gamma_{2}, \quad g_{44}=\eta_{2}+\pi_{22}+\pi_{21}-p_{2} \beta_{2} S_{2}^{*},
\end{gathered}
$$

где

$$
J_{1}=\int_{0}^{\tau} \varphi_{1}(s) d s, \quad J_{2}=\int_{0}^{\tau} \varphi_{2}(s) d s
$$

Предположим, что $G_{\Sigma}$ является невырожденной М-матрицей. Тогда в силу теоремы 1 тривиальное положение равновесия (42) является асимптотически устойчивым.

Отметим, что проверка положительности угловых миноров матрицы $G_{\Sigma}$, включая и положительность ее определителя, приводит к громоздким и плохо обозримым неравенствам. Приведем одно из достаточных соотношений, при выполнении которого $G_{\Sigma}$ является невырожденной М-матрицей. Будем искать $\psi=\left(\psi_{1}, \psi_{2}, \psi_{3}, \psi_{4}\right)^{T}$ такой, что $\psi>0,\left(G_{\Sigma}\right)^{T} \psi>0$. Опираясь на структуру матрицы $\left(G_{\Sigma}\right)^{T}$, положим, что $\psi_{1}=\psi_{3}>0$, $\psi_{2}=\psi_{4}>0$. Тогда искомый $\psi$ существует, если

$$
\begin{aligned}
\min & \left(\frac{\eta_{1}+\lambda_{11}+\pi_{11}-p_{1} \beta_{1} S_{1}^{*}}{\eta_{1}+\left(1-p_{1}\right) \beta_{1} S_{1}^{*}+\rho_{1} J_{1}}, \frac{\eta_{2}+\lambda_{22}+\pi_{22}-p_{2} \beta_{2} S_{2}^{*}}{\eta_{2}+\left(1-p_{2}\right) \beta_{2} S_{2}^{*}+\rho_{2} J_{2}}\right) \\
& >\max \left(\frac{\gamma_{1}}{\lambda_{11}+\gamma_{1}}, \frac{\gamma_{2}}{\lambda_{22}+\gamma_{2}}\right) .
\end{aligned}
$$

Приведенные результаты переносятся на уравнения вида (35)-(41) для трех и более регионов.

\section{Список литературы}

1. Беллман Р. Введение в теорию матрии. М.: Наука, 1976.

2. Воеводин В. В., Кузнецов Ю. А. Матрицы и вычисления. М.: Наука, 1984.

3. Гантмахер Ф. Р. Теория матрии. 2-е изд. М.: Наука, 1966. 
4. Демиденко Г. В., Матвеева И. И. Устойчивость решений дифференциальных уравнений с запаздывающим аргументом и периодическими коэффициентами в линейных членах // Сиб. матем. журн. 2007. T. 48, № 5. C. 1025-1040.

5. Демидович Б. П. Лекиии по математической теории устойчивости. М.: Наука, 1967.

6. Дьери И., Перцев Н. В. Об устойчивости положений равновесия функционально-дифференциальных уравнений запаздывающего типа, обладающих свойством смешанной монотонности // Докл. АН CCCP. 1987. Т. 297, № 1. С. 23-25.

7. Колмановский В. Б. Об устойчивости некоторых систем с произвольным последействием // Докл. РАН. 1993. Т. 331, № 4. С. 421-424.

8. Колмановский В. Б., Носов В. Р. Устойчивость и периодические режимы регулируемых систем с последействием. М.: Наука, 1981.

9. Марчук Г. И. Математические модели в иммунологии. Вычислительные методы и эксперименты. 3-е изд. М.: Наука, 1991.

10. Оболенский А. Ю. Об устойчивости решений автономных систем Важевского с запаздыванием // Укр. матем. журн. 1983. Т. 35, № 5 . C. $574-579$.

11. Перцев Н. В. Глобальная разрешимость и оценки решений задачи Коши для функционально-дифференциальных уравнений с запаздыванием, используемых в моделях живых систем // Cиб. матем. журн. 2018. T. 59, № 1. C. 143-157.

12. Перцев Н. В., Царегородцева Г. Е. Моделирование динамики популяции в условиях воздействия вредных веществ на процесс репродукции особей // Автомат. и телемех. 2011. №1. C. 141-153.

13. Перцев Н. В., Пичугин Б. Ю., Пичугина А. Н. Применение М-матриц для исследования математических моделей живых систем // Mameматическая биология и биоинформатика. 2018. Т. 13, № 2. С. 208-237.

14. Хейл Дж. Теория функционально-дифференциальных уравнений. М.: Мир, 1984.

15. Эльсгольц Л. Э., Норкин С. Б. Введение в теорию дифференииальных уравнений с отклоняющимся аргументом. М.: Наука, 1971.

16. Berman A. and Plemmons R. J. Nonnegative Matrices in the Mathematical Sciences. New York: Academic Press, 1979.

17. Gyori I. Interaction between oscillations and global asymptotic stability in delay differential equations // Differential Integral Equations. 1990. V.3, N 1. P. 181-200. 
18. Kharitonov V. L. Lyapunov functionals and matrices // Annual Reviews in Control. 2010. V. 34. P. 13-20.

19. Medvedeva I. V. and Zhabko A. P. Synthesis of Razumikhin and Lyapunov-Krasovskii approaches to stability analysis of time-delay systems // Automatica. 2015. V.51. P. 372-377.

20. Volz R. Stability conditions for systems of linear nonautonomous delay differential equations // J. Math. Anal. Appl. 1986. V. 120, N 2. P. 584-595.

Перцев Николай Викторович

Институт математики

им. С. Л. Соболева СО РАН

(Омский филиал),

ул. Певцова, 13,

Омск, 644043 РОССИЯ.

E-mail: homlab@ya.ru
Поступила в редакцию 21 октября 2018 г.

Получена после доработки 20 ноября 2018 г.

Принята к публикации 27 февраля 2019 г. 